\title{
3.5-cell large grain niobium superconducting cavity for a dc superconducting rf photoinjector
}

\author{
Shengwen Quan, Feng Zhu, Jiankui Hao, ${ }^{*}$ Xiangyang Lu, Baocheng Zhang, Wencan Xu, and Kui Zhao \\ State Key Laboratory of Nuclear Physics and Technology, Institute of Heavy Ion Physics, Peking University, Beijing, 100871, China
}

(Received 28 November 2009; published 30 April 2010)

\begin{abstract}
We have developed a new compact high average current dc superconducting rf (DC-SRF) photoinjector. A 3.5-cell superconducting cavity with a mid-beta structure is the key component of the DC-SRF photoinjector. Herein, we present the new design, fabrication, and vertical test results of the 3.5-cell large grain niobium superconducting cavity. After preliminary surface preparation and room temperature rf measurements, the cavity was sent to Jefferson Lab for a vertical cold test. The gradient of the cavity reaches $23.5 \mathrm{MV} / \mathrm{m}$, and the $Q_{0}$ value is higher than $1.2 \times 10^{10}$. The high-quality 3.5-cell cavity will guarantee the good performance of the DC-SRF photoinjector.
\end{abstract}

DOI: 10.1103/PhysRevSTAB.13.042001

PACS numbers: 74.25.N-, 84.40.- $-\mathrm{x}, 81.65 . \mathrm{Cf}$

\section{INTRODUCTION}

A high average current electron gun with high brilliance is absolutely essential for future free-electron lasers (FEL), light sources, and high energy linear accelerators. The concept of combining of a Pierce gun with a superconducting cavity was first proposed by our group at Peking University (PKU) in 2001 [1]. A prototype photoinjector with a Pierce gun and a $1+1 / 2$ cell superconducting cavity was first manufactured in 2002; feasibility was demonstrated. The photocathode is placed on the cathode of the Pierce structure; the Pierce anode is located at the entrance of the superconducting cavity. With this design, we solved the compatibility problem between the superconducting cavity and the photocathode. Then, we demonstrated the $\mathrm{dc}$ superconducting $\mathrm{rf}$ (DC-SRF) photoinjector in 2004 [2].

The Peking University Free Electron Laser (PKU-FEL) facility is built and operated by the PKU SRF laboratory. The PKU-FEL will run in the infrared (5-10 $\mu \mathrm{m})$ and THz $(100-3000 \mu \mathrm{m})$ region with high stability and high average power. The DC-SRF photoinjector is the electron source for the PKU-FEL. Based on the results of the prototype injector, we are developing a new DC-SRF photoinjector. The new photoinjector provides electron beam with low emittance at $1.2 \mathrm{~mm}$ mrad, high average current at $1 \mathrm{~mA}$, and short bunch length at several picoseconds. The injector combines a dc pierce gun and a 3.5cell superconducting cavity. It allows $\mathrm{cw}$ operation or long pulsed mode operation with high repetition rate, and thus produces high average current. The design energy of the injector is $5 \mathrm{MeV}$. Figure 1 shows a three-dimensional schematic of the injector.

The beam quality of the injector is mainly determined by the performance of the 3.5-cell superconducting cavity.

\footnotetext{
*Corresponding author. jkhao@pku.edu.cn
}

Thus, intensive research has been made to improve the performance of the cavity.

In the following, we report the design, fabrication, surface treatment, and cold test of the new 3.5-cell SC cavity.

\section{DESIGN OF THE 3.5-CELL CAVITY}

\section{A. Geometry optimization}

The core components of the new DC-SRF injector are a $100 \mathrm{kV}$ dc pierce gun and a 3.5-cell superconducting cavity. Compared to the Rossendorf SRF gun cavity [3], our cavity has no choke filter structure. After dc acceleration, the electrons emitted from the photocathode go directly into the 3.5-cell superconducting cavity. Figure 2 shows the key components of the injector.

The optimization of the first cell is critical to guarantee the properties of the electron beam. The last three cells of the 3.5-cell superconducting cavity adopt the geometry of a TESLA mid-cup and end cup [4]. During optimization of the cavity main emphasis was put on the first half cell. The first half cell connects the anode of the dc pierce structure with a small $12 \mathrm{~mm}$ diameter beam tube. In order to

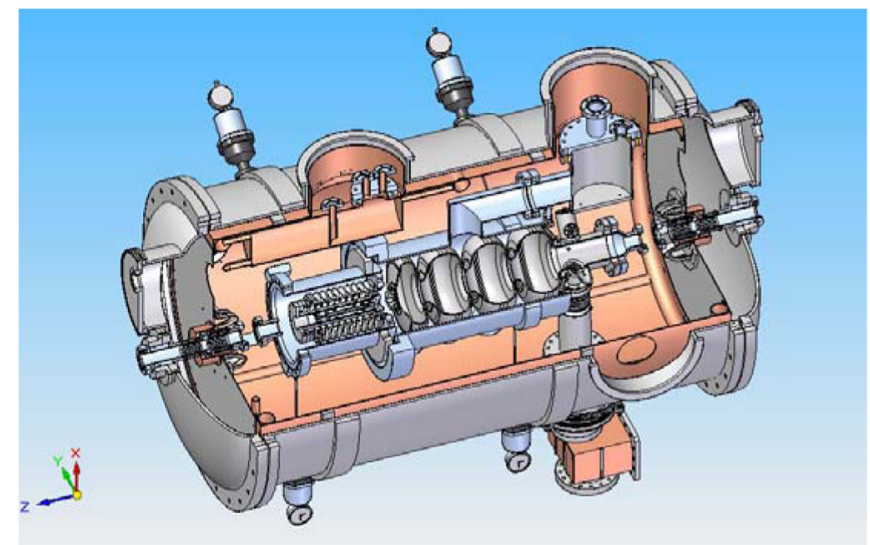

FIG. 1. (Color) Schematic design of 3.5-cell DC-SRF photoinjector at PKU. 


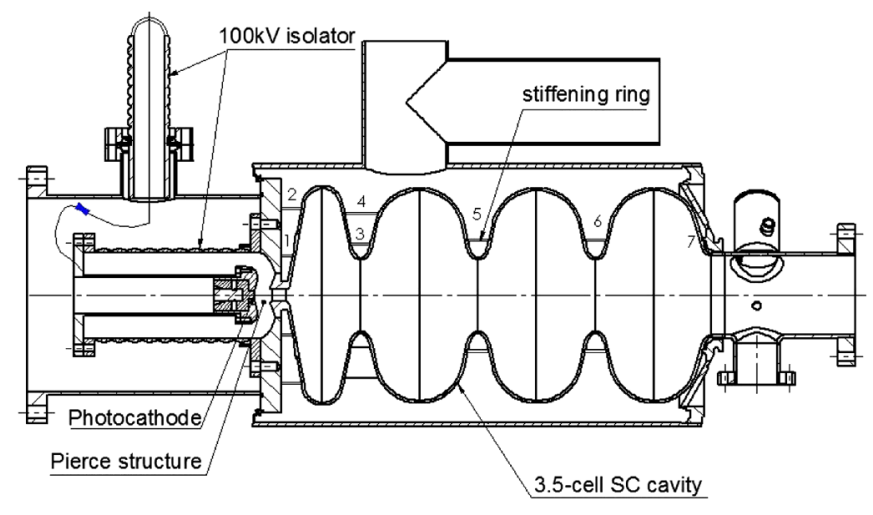

FIG. 2. Key components of the DC-SRF injector.

decrease the emittance growth, the length of this small beam tube is designed to be as short as possible. To prevent the fields of the superconducting cavity from going into the $\mathrm{dc}$ structure, the tube is designed at $17 \mathrm{~mm}$ long. We also made beam dynamics simulations to assure the good matching between the dc structure and the 3.5-cell cavity. The cavity is optimized to low $E_{p} / E_{\text {acc }}$ and $B_{p} / E_{\text {acc }}$. The geometry beta of the first half cell is 0.63 . With a conical wall of $10.5^{\circ}$, the first half cell has a negative value of the radial field near the entrance of the cavity. It focuses the electron beam after the dc structure at an energy of $90 \mathrm{keV}$. The reasons that the conical wall is designed with such an angle are: (1) to avoid field emission with a better cavity cleaning effect; and (2) to achieve better mechanical prop-

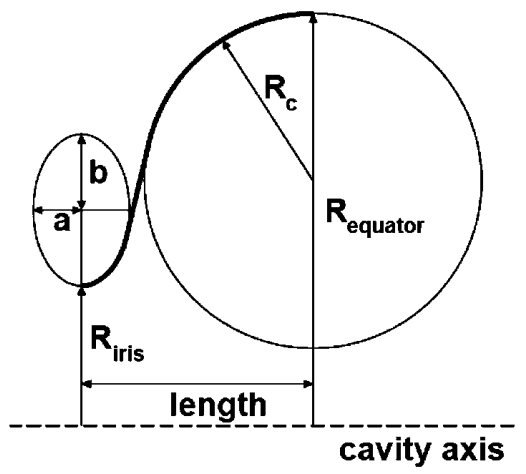

FIG. 3. Geometry of the 3.5-cell cavity.

TABLE I. Geometry parameters of the 3.5-cell cavity (unit: $\mathrm{mm})$.

\begin{tabular}{lcccc}
\hline \hline & Mid-cell & $\begin{array}{l}\text { Left cup } \\
\text { (1st cell) }\end{array}$ & $\begin{array}{l}\text { Right cup } \\
\text { (1st cell) }\end{array}$ & $\begin{array}{c}\text { End } \\
\text { cup }\end{array}$ \\
\hline$R_{\text {equator }}$ & 103.3 & 105.3 & 105.3 & 103.3 \\
$R_{\text {iris }}$ & 35 & 6 & 35 & 39 \\
$R_{c}$ & 42.0 & 17.14 & 17.14 & 40.3 \\
$a$ & 12 & 3 & 12 & 10 \\
$b$ & 19 & 3 & 20 & 13.5 \\
Length & 57.7 & 35.19 & 37.72 & 56.0 \\
\hline \hline
\end{tabular}

TABLE II. Design parameters of the 3.5-cell cavity.

\begin{tabular}{lc}
\hline \hline Mode & $\mathrm{TM}_{010}, \pi$ mode \\
\hline Frequency $(\mathrm{MHz})$ & 1300 \\
$Q_{0}$ & $1 \times 10^{10}$ \\
$E_{\text {acc }}(\mathrm{MV} / \mathrm{m})$ & 15 \\
Effective length $(\mathrm{m})$ & 0.417 \\
$\mathrm{G}$ factor $(\Omega)$ & 242 \\
$E_{\text {peak }} / E_{\text {acc }}$ & 2.12 \\
$B_{\text {peak }} / E_{\text {acc }}(\mathrm{mT} /(\mathrm{MV} / \mathrm{m}))$ & 4.95 \\
$r / Q(\Omega)$ & 417 \\
\hline \hline
\end{tabular}

erties. Figure 3 depicts the geometry of the 3.5-cell cavity; Table I shows the optimized parameters of the 3.5-cell cavity. Table II shows the rf parameters of the cavity.

\section{B. Multipacting simulation}

We made the multipacting simulations for the 3.5-cell SC cavity with the code FISHPACT [5]. Figure 4 shows the electron trajectory orbit and enhanced counter function, respectively. The results indicate that the multipacting barrier can be processed for good cavity surface.

\section{Lorentz force detuning}

The bandwidth of the 3.5-cell cavity is only about $130 \mathrm{~Hz}$ since the external $Q$ value $\left(Q_{e}\right)$ is about $1 \times 10^{7}$. The frequency shift caused by the Lorentz force detuning is higher than $500 \mathrm{~Hz}$ when the cavity is operated at $13 \mathrm{MV} / \mathrm{m}$ (provided that there are no stiffening rings). Stiffening rings are designed and added to the cavity. By

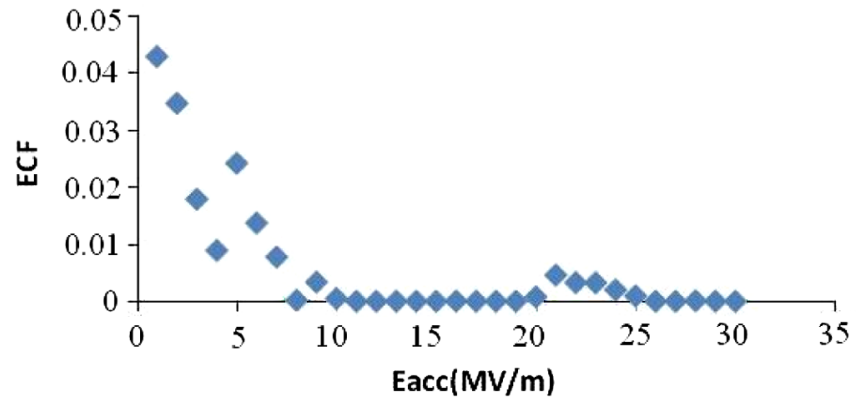

(a)

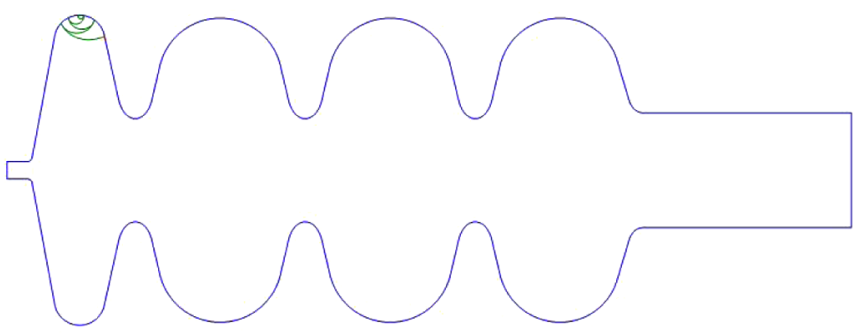

(b)

FIG. 4. (Color) Multipacting simulation (a) enhanced counter function. (b) Electron trajectory orbit at the first half cell. 
optimization, the Lorentz force detuning coefficient is reduced to $1.2 \mathrm{~Hz} /(\mathrm{MV} / \mathrm{m})^{2}$.

\section{Cross talk}

Because of the dc structure, the two higher order mode (HOM) couplers, the main power coupler port, and the pickup port are located at the same end of the 3.5-cell cavity. Therefore a direct coupling between the power coupler and the pickup probe has to be investigated. Such a cross talk should be avoided in order to not disturb the low level $\mathrm{rf}$ control of the injector. Calculation and $\mathrm{rf}$ measurements were made with a two-cell copper cavity. The results showed that the transmitted power to the pickup from the cavity is 6 orders higher than the one from the main coupler at superconducting conditions. Thus, the cross talk between the pickup antenna and the main power coupler can be neglected for the injector operation.

\section{3.5-CELL LARGE GRAIN NIOBIUM CAVITY FABRICATION}

In order to assure the high-level performance, we have made studies on the large grain niobium superconducting cavities at Peking University since 2005. A $1.3 \mathrm{GHz}$ singlecell and a two-cell TESLA-type cavity were fabricated with Ningxia (China) large grain niobium sheets since then. Afterwards, the two cavities were tested at Jlab (U.S.A.) and DESY (Germany), respectively. Both cavities show excellent results [6-8]: gradients are higher than $40 \mathrm{MV} / \mathrm{m}$ at a $Q$ value above $1 \times 10^{10}$.

Because of the complex structure, the 3.5-cell cavity is difficult to pursue electrical polishing. Based on the nice results of the large grain single-cell and two-cell cavities, we choose the Ningxia large grain niobium sheets to fabricate the 3.5-cell cavity, which guarantees the performance by buffered chemical polishing (BCP). A single crystal niobium sheet with a diameter of $70 \mathrm{~mm}$ is used to make the anode and the entrance of the first half cell. This avoids discharging at high surface field.

Electron beam welding of the 3.5-cell cavity was finished at Harbin Institute of Technology, China. Quality control and rf measurements were made at Peking University. After the leak check, mechanical measurements and warm $\mathrm{rf}$ measurements were performed at Peking University. Then, the cavity went through $1250^{\circ} \mathrm{C}$ post-purification and $\mathrm{BCP}$ treatment at Ningxia. Figure 5 shows the photograph of the 3.5-cell cavity.

In order to achieve high gradient, the cavity needs field flatness tuning at room temperature to reach the right field distribution. According to the design, the field of the half cell is identical with the other three cells. We made the warm rf measurement and the field flatness tuning at the multicell cavity tuning facility at Peking University. The field flatness of the cavity after tuning is $94 \%$. We did not make any further adjustment since the cavity would be sent

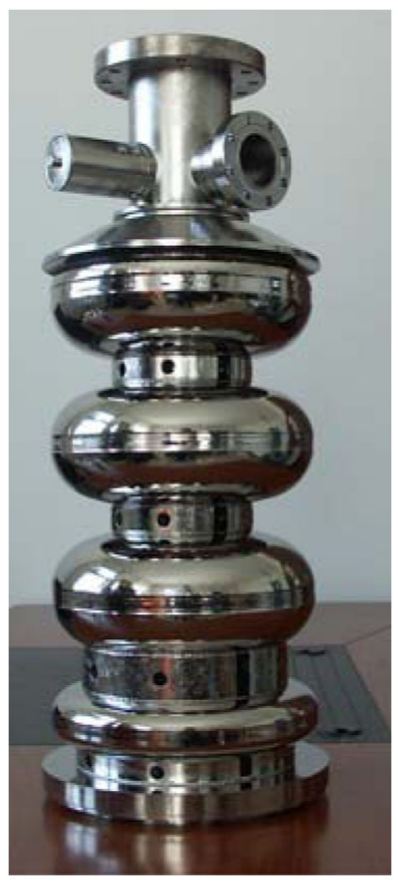

FIG. 5. (Color) 3.5-cell large grain niobium cavity after $1250^{\circ} \mathrm{C}$ post-purification and BCP at Ningxia.

to Jlab for vertical test. The lowest field was measured in the first cell. The frequency of the $\pi$ mode is $1294.83 \mathrm{MHz}$.

When the injector is in operation, the HOMs dissipated power is less than $1 \mathrm{~W}$. Therefore we choose TESLA-type HOM couplers. Figure 6 shows the passband properties of HOM couplers welded on the 3.5-cell cavity. We can see that there is no effect to the fundamental $\mathrm{TM}_{010}$ mode while the HOMs can be extracted effectively.

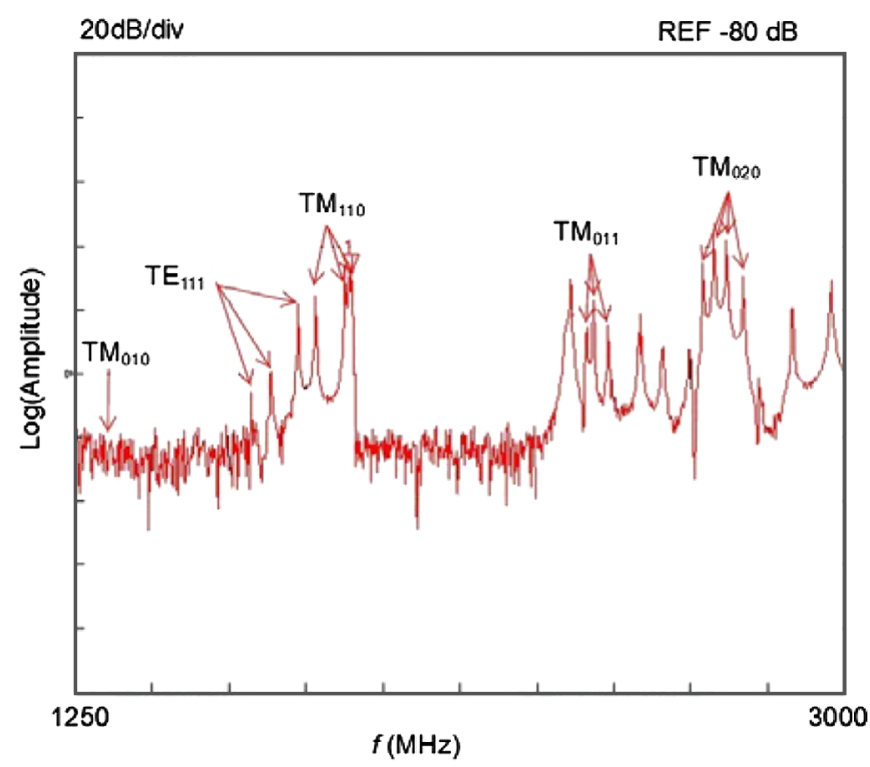

FIG. 6. (Color) Properties of HOM couplers of the 3.5-cell cavity. $\mathrm{TM}_{010}$ mode is cut off and the HOMs can be extracted effectively. 


\section{VERTICAL COLD TEST}

After $\mathrm{rf}$ test and field flatness tuning, the $100 \mu \mathrm{m}$ surface of the 3.5-cell large grain niobium cavity was etched by $\mathrm{BCP}$ treatment followed by a three-hour postpurification under $1250^{\circ} \mathrm{C}$ at Ningxia OTIC. After a flash $\mathrm{BCP}$ and high pressure rinsing (HPR), the cavity was sent to Jlab for vertical test.

Because of the special structure of the 3.5-cell cavity, Dr. Rong-Li Geng helped perform further treatment in Jlab. After delivery to Jlab, the first and second cold tests of the 3.5-cell cavity were made after HPR only. The gradient of the cavity was only $7 \mathrm{MV} / \mathrm{m}$, limited by strong field emission. New BCP treatment and HPR cleaning in a specially optimized system were applied for the 3.5-cell cavity. $45^{\circ}$ nozzles were added based on the standard ninecell cavity HPR system. After $30 \mu \mathrm{m} \mathrm{BCP}$ removal and new HPR at Jlab, the third cold test of the cavity was done. There was no field emission at this time, and the gradient increased to $11 \mathrm{MV} / \mathrm{m}$, limited by strong multipacting, which occurred from $8 \mathrm{MV} / \mathrm{m} . Q_{0}$ is only $6 \times 10^{9}$ even at low field, see Fig. 7.

Multipacting simulation of the 3.5-cell cavity predicts two possible regions for multipacting. One region is lower than $10 \mathrm{MV} / \mathrm{m}$, referring to the first half cell. The other is $18-23 \mathrm{MV} / \mathrm{m}$, responding to the three TESLA-type cells, see Fig. 4. The barriers are not so strong. The low $Q_{0}$ means that the surface of the cavity was polluted during BCP due to the small beam hole. In order to cure the $Q$ drop and eliminate the multipacting, $800^{\circ} \mathrm{C}$ heat treatment and additional $30 \mu \mathrm{m}$ BCP were made followed by HPR. During the first power rise of the 4th test, two multipacting barriers occurred at $8 \mathrm{MV} / \mathrm{m}$ and $18 \mathrm{MV} / \mathrm{m}$ and were processed finally. The gradient reaches $23.5 \mathrm{MV} / \mathrm{m}$, and the $Q$ value is above $1.2 \times 10^{10}$, limited by quench, see Fig. 8 . The performance of the 3.5-cell large grain cavity is better than the designed value. This is very helpful in order to improve the performance of DC-SRF photoinjector operation.

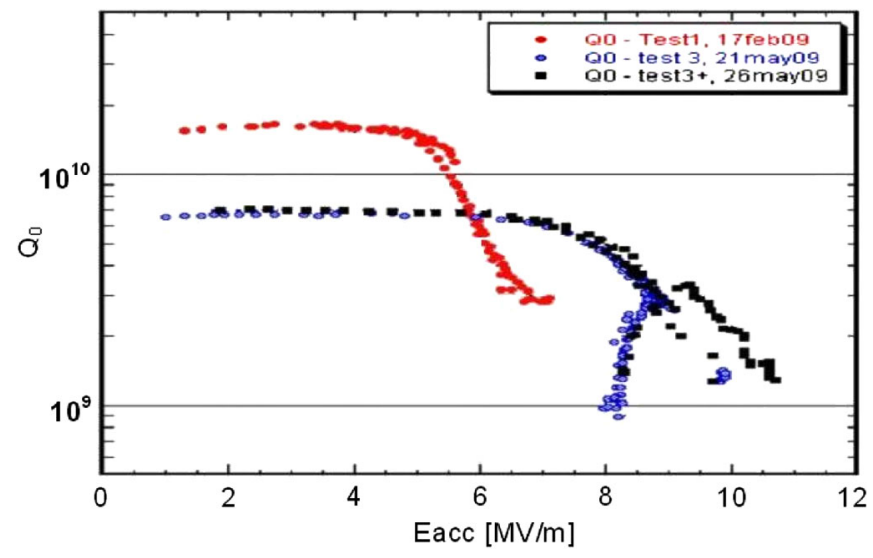

FIG. 7. (Color) $Q_{0}$ vs $E_{\text {acc }}$ was measured for the 3.5-cell cavity. Test 1 was done after HPR at Jlab only (red dot). Test 3 was performed after $30 \mu \mathrm{m} \mathrm{BCP}$ and HPR (blue circle) and warm-up to room temperature then cooldown (black square).

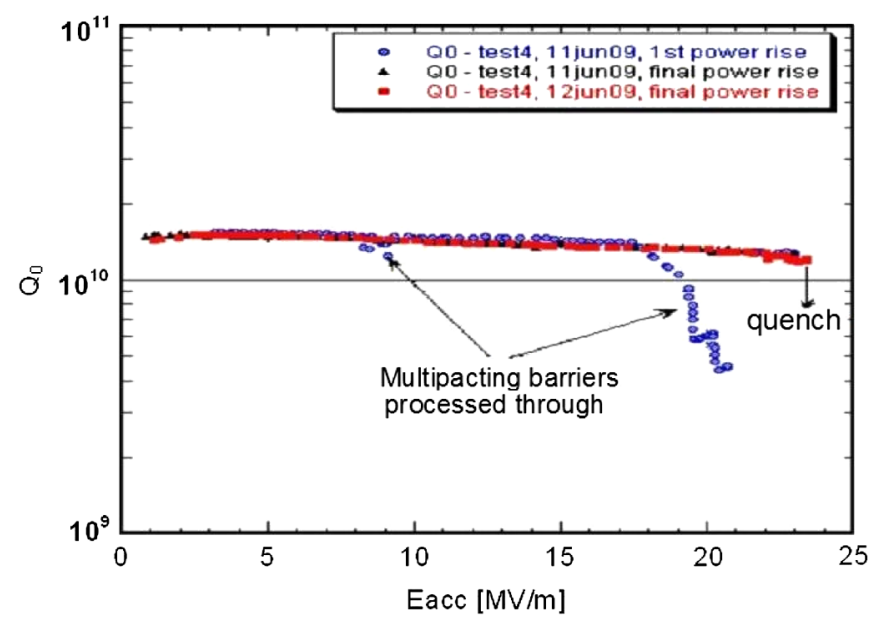

FIG. 8. (Color) Final test result of 3.5-cell large grain cavity after $800^{\circ} \mathrm{C}$ heat treatment and additional $30 \mu \mathrm{m} \mathrm{BCP}$ followed by HPR. Multipacting barriers occurred and were processed during the 1st power rise (blue circle). Gradient reached 23.5 MV/m for the final power rise (black triangle and red square).

\section{CONCLUSION}

A well performing 3.5-cell cavity was designed by the SRF group at Peking University for the DC-SRF photoinjector. Field flatness tuning, Lorentz force detuning, HOM extraction, and cross talk were properly solved by optimization, calculation, and rf measurements. A large grain 3.5-cell cavity was fabricated with China Ningxia niobium at Peking University. The 3.5-cell large grain niobium cavity was $\mathrm{BCP}$ etched and $1250^{\circ} \mathrm{C}$ purified with titanium protection in China. After field flatness tuning, the cavity was sent to Jlab for further treatment and vertical test. The accelerating gradient of the 3.5-cell cavity finally reaches $23.5 \mathrm{MV} / \mathrm{m}$ and the $Q_{0}$ is above $1.2 \times$ $10^{10}$. This is very important for good performance of the DC-SRF photoinjector. The 3.5-cell large grain niobium cavity was welded to the liquid helium tank and then installed at the new DC-SRF photoinjector. The 3.5-cell cavity will be tested with the cryomodule for the next step.

\section{ACKNOWLEDGMENTS}

The authors are grateful to Ningxia OTIC colleagues for the cavity treatment, Professor J. He at Harbin Institute of Technology for the cavity welding, and Dr. Rong-Li Geng at Jlab for the cavity treatments and testing. This work is supported by the Major State Basic Research Development Program of China under Grants No. 2002CB713602 and No. 2008CB817706.

[1] Kui Zhao, Jiankui Hao, Yanle Hu, Baocheng Zhang, Shengwen Quan, Jiaer Chen, and Jiejia Zhuang, Nucl. Instrum. Methods Phys. Res., Sect. A 475, 564 (2001). 
[2] Jiankui Hao, Xiangyang Lu, Yuantao Ding, Shengwen Quan, Senlin Huang, Kui Zhao, Baocheng Zhang, Lifang Wang, Lin Lin, Fei Jiao, Guimei Wang, Datao Xie, Feng Zhu, Binping Xiao, Rong Xiang, and Jia'er Chen, Nucl. Instrum. Methods Phys. Res., Sect. A 557, 138 (2006).

[3] B. Aune et al., Phys. Rev. ST Accel. Beams 3, 092001 (2000).

[4] P. Murcek et al., in Proceedings of the 14th Workshop on RF Superconductivity, Berlin, Germany, 2009, http:// accelconf.web.cern.ch/AccelConf/srf2009/, p. 585.
[5] http://www.jlab.org/ genfa/fishpact.

[6] Chen Jia-er and Zhao Kui, in Proceedings of the 13th Workshop on RF Superconductivity, Beijing, China, 2007, http://accelconf.web.cern.ch/AccelConf/srf2007/, p. 1.

[7] Hao Jian-Kui, Zhao Kui, Zhang Bao-Cheng, Quan ShengWen, Wang Li-Fang, and Chen Jia-Er, Chinese Phys. C 32, 181 (2008).

[8] Krzysztof Twarowski and D. Reschke (private communication). 